\title{
Bacterial Profile of Urinary Tract Infection in Type 2 Diabetes Mellitus
}

\author{
Sithara Chunakkara Jayadeswari ${ }^{1}$, Sreekumary Puthusseril Kunjappan² \\ ${ }^{1}$ Department of Microbiology, Government Medical College, Kottayam, Kerala, India. \\ ${ }^{2}$ Department of Microbiology, Government Medical College, Kottayam, Kerala, India.
}

\section{ABSTRACT}

\section{BACKGROUND}

Diabetes mellitus is one of the most challenging heath problems of the $21^{\text {st }}$ century and is leading cause of death in developed countries. Urinary tract infections are one of the most frequent bacterial infections encountered in diabetes mellitus. We wanted to determine the common pathogens causing urinary tract infection in diabetic patients and their antibiotic resistance pattern.

\section{METHODS}

A total of 550 mid-stream clean catched urine samples were subjected to macroscopic examination, wet film examination, Gram staining, and semi-quantitative culture methods. HiCrome agar was used for early identification of isolates. Samples were inoculated on 5\% blood agar, CLED agar and MacConkey agar. Isolates were identified by standard laboratory procedures.

\section{RESULTS}

In this study, 284 isolates were obtained, and the most common organisms isolated were E. coli. Klebsiella and Proteus species. Gram positive isolates were Enterococcus and MRSA. Patients with diabetes are more prone to have resistant pathogen.

\section{CONCLUSIONS}

This study concluded that the prevalence of urinary tract infection is higher in females compared to males in diabetes. Gram stain was found to be the best screening test with high sesitivity and specificity. Wet film has comparatively low sesitivity and specificity. It is essential that culture should be done for all samples in diabetes to decrease the morbidity and prevent the occurrence of complications.
Corresponding Author:

Dr. Sreekumary Puthusseril Kunjappan, Additional Professor of Microbiology,

Government Medical College,

Kottayam, Gandhinagar-686008,

Kerala, India.

E-mail: drsreekumarypk@gmail.com

DOI: $10.14260 /$ jemds/2020/524

How to Cite This Article:

Jayadeswari SC, Kunjappan SP. Bacterial profile of Urinary tract infection in Type 2 Diabetes mellitus. J Evolution Med Dent Sci 2020;9(34):2407-2412,

$10.14260 /$ jemds/2020/524

Submission 28-03-2020,

Peer Review 13-07-2020,

Acceptance 20-07-2020,

Published 24-08-2020.

Copyright (C) 2020 JEMDS. This is an open access article distributed under Creative Commons Attribution License [Attribution 4.0 International (CC BY 4.0)]

\section{KEY WORDS}

Diabetes Mellitus, E. coli, Klebsiella, HiCrome Agar, UTI 


\section{BACKGROUND}

The incidence of diabetes mellitus throughout the world is increasing and is becoming a public health problem especially in developing countries. Diabetes mellitus is a group of metabolic disorder characterised by increased blood glucose level resulting from defects in insulin secretion, insulin action or both. ${ }^{1}$ longer duration of diabetes with uncontrolled hyperglycaemia is associated with the prevalence of bacteriuria. Type 2 diabetes mellitus is characterized by impaired insulin secretion, insulin resistance, excessive hepatic glucose production, and abnormal fat metabolism. ${ }^{2}$ Urinary tract infections are one of the most common infections occurring both in community and hospital patients especially in diabetics. It is a frequent cause of morbidity, mortality and major cause of antibiotic resistance. The most common isolate is Escherichia coli and other reported organisms are Proteus, Klebsiella, Enterococcus, Pseudomonas, Staphylococcus, Candida albicans. ${ }^{1}$ Urinary tract infection can be symptomatic or asymptomatic. Symptomatic urinary tract infections are characterised by symptoms like dysuria, urgency, painful and frequent voiding, accompanied by the presence of bacteria in the sterile urine. Asymptomatic bacteriuria is defined as persistently and actively multiplying bacteria in significant numbers i.e., $10^{5}$ bacteria per $\mathrm{ml}$ within the urinary tract without any obvious symptoms.

Urinary tract infection occurs as a result of interaction of bacterial virulence and host biologic and behavioural factors as opposed to highly efficient host defence mechanisms. Patient with diabetes have a 10-fold increased risk of Significant bacteriuria Proof of urinary tract infection requires the demonstration that the potential pathogens present in freshly voided urine in numbers greater than those likely to result from contamination from urethral meatus and its environs. The observations of Kass (1957) suggested that this number taken to indicate significant bacteriuria is about $100000 \mathrm{CFU} / \mathrm{mL}$. Significant bacteriuria is defined as the presence of more than $10^{5}$ colony forming units per ml or $>10^{8}$ organisms per litre in a midstream clean catch urine sample which is promptly transported and processed.

Urinary tract infection occurs with increased frequency and severity in patients with diabetes mellitus. Longer duration of diabetes with uncontrolled hyperglycaemia is associated with the prevalence of bacteriuria. General risk factors for urinary tract infection in diabetes mellitus include age, metabolic control, and long-term complications primarily diabetic nephropathy and cystopathy. Diabetes cause several abnormalities of the host immune system which results in high risk of urinary tract infection.$^{1}$ Patients with diabetes have a 10 -fold increased risk of urinary tract infection when compared to non- diabetes. ${ }^{3}$ Early diagnosis and prompt intervention is recommended to limit morbidity of symptomatic infection. From a microbial perspective urinary tract infection exists when pathogenic microorganisms are detected in the urine, urethra, bladder, kidney, or prostate with or without symptoms. Urinary tract infection may involve only the lower urinary tract or both the upper and lower tracts. Uncomplicated urinary tract refers to infection in a structurally and neurologically normal urinary tract. Complicated urinary tract infection refers to infection in a urinary tract with functional or structural abnormalities, including indwelling catheters and calculi.

\section{Objectives}

1. To identify the bacterial pathogens causing urinary tract infection in subjects with type 2 diabetes mellitus.

2. To describe the antibiotic sensitivity pattern of the bacterial isolates.

\section{METHODS}

This study was conducted in Government Medical College, Kottayam, for a period of one year from May 2017 to April 2018.

\section{Inclusion Criteria}

- $\quad$ Type 2 diabetes patients

- More than 18 years of age

- Presenting with clinical features of urinary tract infection.

\section{Exclusion Criteria}

- Patients on antibiotics or those who have taken antibiotics within 2 weeks.

- Age less than 18 years.

- Non-diabetic patients.

- Type 1 diabetics.

\section{Sample Size and Methodology}

550 is the minimum sample size as calculated with formula $4 \mathrm{pq} / \mathrm{d}^{2}$ using prevalence (p) ${ }^{2}$ and Non-probability convenient sampling method.

\section{Definitions and Formulae}

True positive (TP): Screening test and culture, both positive. False positive (FP): Positive scree binning test and culture, both negative.

True negative (TN): Screening test and culture, both negative. False negative (FN): Screening test negative and culture positive.

Sensitivity $=\mathrm{TP} /(\mathrm{TP}+\mathrm{FN})$ : Probability that screening test will be positive in patients with UTIs (Positive Culture).

Specificity $=$ TN $(\mathrm{TN}+$ FP): Probability that screening test will be negative in patients without UTIs (Negative Culture).

Positive predictive value (PPV): TP / (TP + FP): Probability that a UTI is present when screening test is positive.

Negative predictive value (NPV): TN/ $(\mathrm{TN}+\mathrm{FN})$ : Probability that a UTI is not present when screening test is negative.

\section{Sample Collection and Processing}

A total of 550 samples were collected from patients diagnosed to have diabetes mellitus presenting with features of urinary tract infection, after taking informed consent. The patients were given instructions on how to collect sample. The samples were taken in a sterile leak proof universal container and immediately transferred and processed within 2 hours of collection in the laboratory. Samples were subjected to macroscopic examination, wet film examination, Gram stain 
and semi - quantitative culture method. The semi-quantitative culture was done by surface streaking method on $5 \%$ sheep blood agar, Cysteine Lactose Electrolyte Deficient agar with bromo-thymol blue and MacConkey agar. Hi Chrome UTI agar was used for early identification of isolates. Sample was inoculated and incubated at $37^{\circ} \mathrm{C}$ overnight.

\section{HiCrome UTI Agar - Different Colour of Bacteria}

- E. coli - dark pink to reddish.

- Proteus - brown halo.

- Klebsiella - metallic blue.

- $\quad$ S. aureus - Golden, Opaque, Small

- P. aeruginosa - translucent, cream to blue.

\section{Screening Tests Done}

Wet Film Examination of Un-Centrifuged Urine - Finding of more than 1 leukocyte per 7 high power fields corresponds with more than $10^{4}$ leukocytes per ml which was considered as significant pyuria.

Gram Stain of Well Mixed Un-Centrifuged Urine - The stained smear was examined and the presence of at least one bacterium per oil immersion field in a clean catched midstream urine co-relates with $10^{5}$ bacteria per ml of urine or more. Smear was discarded as negative after examining at least 20 oil immersion fields. ${ }^{3}$

Semi-Quantitative Culture of Urine - The calibrated loop was introduced vertically into the thoroughly mixed urine ${ }^{4}$ and taken out. Loop was touched to the centre of the plate, from which the inoculum was spread in a line across the plate. Without flaming or re-entering urine, loop was drawn across the entire plate, crossing the first inoculum streak numerous times to produce isolated colonies. Plates were incubated at $37^{\circ} \mathrm{C}$ for 24 hours. Number of colonies were counted and multiplied by 1000 to get the colony count.

\section{Identification of Isolates}

The identification of the bacterial pathogens was done by staining, cultural and biochemical properties using standard laboratory procedures. $2,4,5$

\section{Antibiotic Sensitivity Testing of Isolate}

Antibiotic sensitivity testing was done using Kirby- Bauer method with 0.5 McFarland standard turbidity of the inoculum. Standard strains of Escherichia coli (ATCC 25922), Enterococcus faecalis (ATCC 29212) and Staphylococcus aureus (ATCC 25923) were used as control. Antibiotics were tested and zone of inhibition of growth was measured and interpreted according to CLSI guidelines M100. ${ }^{6}$ All antibiotics were not reported. Antibiotics which can be given orally were preferred for reporting. For gram-negative isolates Ampicillin (25 mcg), Gentamicin, Cephalosporin (1 1 st, $3^{\text {rd }}$ generation), Ciprofloxacin, Norfloxacin, Nitrofurantoin, Amikacin, Trimethoprim-Sulfamethoxazole, Piperacillin-
Tazobactam, Cefoperazone-Sulbactum and Meropenem. For Staphylococcus spp. - Ampicillin, Gentamicin, First generation Cephalosporin, Amikacin, Cefoxitin and Vancomycin.

Test for Extended Spectrum Beta-Lactamase Production ${ }^{6}$ Those isolate with Cefotaxime (30 mcg) zone less than $27 \mathrm{~mm}$ after incubating in Mueller-Hinton agar at $37^{\circ} \mathrm{C}$ overnight was selected for doing confirmatory test for ESBL production. Ceftazidime, Ceftazidime-Clavulanic acid disc were placed in Mueller-Hinton agar after doing lawn culture of the strain and incubated overnight at $37{ }^{\circ} \mathrm{C}$. A>5 mm increase in zone diameter for either antimicrobials tested in combination with Clavulanate versus the zone diameter of the agent tested alone confirms ESBL production. Two gram-positive budding yeast cell was also isolated, identified as Candida tropicalis by negative germ tube test, on Corn Meal agar - Abundant long, branching pseudohyphae. Ovoid or elongate blastoconidia are found along the pseudohyphae, including adjacent to the junctions. Urea not hydrolysed. Sabouraud's Dextrose broth (SDA) - surface film, with bubbles. ${ }^{6}$

\section{Analysis and Interpretation}

The data was numerically coded and was entered in Microsoft excel spread sheet. Further analysis was done using the software SPSS and tables and charts were created using excel.

\begin{tabular}{|c|c|c|}
\hline \multicolumn{3}{|c|}{ RESULTS } \\
\hline Gender & Sample Number & Culture Positive \\
\hline Male & 252 & 136 \\
\hline Female & 298 & 148 \\
\hline Total & 550 & 284 \\
\hline \multicolumn{3}{|c|}{ Table 1. Overview of Samples } \\
\hline
\end{tabular}

\begin{tabular}{|cccc|}
\hline Macroscopy & Absent & Present & Total \\
Clear & 209 & 63 & 272 \\
Slightly turbid & 32 & 53 & 85 \\
Turbid & 25 & 168 & 193 \\
Total & $\mathbf{2 6 6}$ & $\mathbf{2 8 4}$ & $\mathbf{5 5 0}$ \\
\hline Table 2. Association between Macroscopy and Significant Bacteriuria \\
\hline
\end{tabular}

\begin{tabular}{|ccccc|}
\hline \multirow{4}{*}{ Wet Film } & Presence of Pus Cells & \multicolumn{2}{c|}{ Isolate } & \multirow{2}{*}{ Total } \\
& and Bacteria & Absent & Present & \\
& No pus cells, No Bacteria & 210 & 60 & 270 \\
& Pus cells < 5, Bacteria+ & 55 & 211 & 266 \\
& Pus cells > 5, Bacteria+ & 1 & 13 & 14 \\
& Total & $\mathbf{2 6 6}$ & $\mathbf{2 8 4}$ & $\mathbf{5 5 0}$ \\
\hline Table 3. Association between Pyuria and Significant Bacteriuria \\
\hline
\end{tabular}

\begin{tabular}{|ccc|}
\hline Isolates & Frequent & Percentage \\
Gram negative organisms & $\mathbf{2 7 0}$ & $\mathbf{9 5 . 1}$ \\
E. coli & 134 & 47.2 \\
Klebsiella & 80 & 28.2 \\
Pseudomonas & 24 & 8.5 \\
Acinetobacter & 18 & 6.3 \\
Proteus & 10 & 3.5 \\
Citrobacter & 4 & 1.4 \\
Gram positive organisms & $\mathbf{1 4}$ & $\mathbf{4 . 9}$ \\
Enterococcus & 13 & 4.6 \\
MRSA & 1 & 0.4 \\
Total & $\mathbf{2 8 4}$ & $\mathbf{1 0 0}$ \\
\hline \multicolumn{2}{c}{ in Diable 4. Microbiological Profile of Significant Bacteriuria } \\
\hline
\end{tabular}




\begin{tabular}{|c|c|c|c|c|c|c|}
\hline$\frac{\stackrel{\mathscr{U}}{\pi}}{\frac{\pi}{0}}$ & 뭉 & 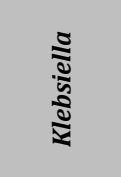 & 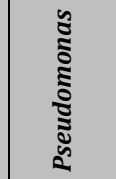 & 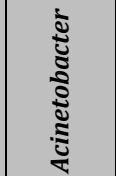 & $\begin{array}{l}\frac{\Xi}{0} \\
\vdots \\
\vdots\end{array}$ & 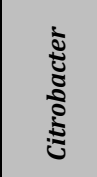 \\
\hline Ampicillin & $0(0 \%)$ & $0(0 \%)$ & $0(0 \%)$ & $0(0 \%)$ & $0(0 \%)$ & $0(0 \%)$ \\
\hline Gentamicin & $25(18.7 \%)$ & $14(17.5 \%)$ & $6(25 \%)$ & $5(27.8 \%)$ & $2(20 \%)$ & $0(0 \%)$ \\
\hline Erythromycin & $0(0 \%)$ & $0(0 \%)$ & $0(0 \%)$ & $0(0 \%)$ & $0(0 \%)$ & $0(0 \%)$ \\
\hline \begin{tabular}{|c|} 
Cephalexin \\
\end{tabular} & $5(3.7 \%)$ & $4(5 \%)$ & $0(0 \%)$ & $0(0 \%)$ & $2(20 \%)$ & $0(0 \%)$ \\
\hline Amikacin & $82(61.2 \%)$ & $44(55 \%)$ & $14(58.3 \%)$ & $9(50 \%)$ & $3(30 \%)$ & $2(50 \%)$ \\
\hline Cotrimoxazole & $69(51.5 \%)$ & $41(51.3 \%)$ & $0(0 \%)$ & $6(33.3 \%)$ & $7(70 \%)$ & $2(50 \%)$ \\
\hline Ciprofloxacin & $16(11.9 \%)$ & $15(18.8 \%)$ & $8(33.3 \%)$ & $2(11.1 \%)$ & $2(20 \%)$ & $0(0 \%)$ \\
\hline \begin{tabular}{|l|} 
Cefotaxime \\
\end{tabular} & $17(12.7 \%)$ & $12(15 \%)$ & $0(0 \%)$ & $3(16.7 \%)$ & $2(20 \%)$ & $1(25 \%)$ \\
\hline \begin{tabular}{|c|} 
Cefoperazone- \\
Sulbactum
\end{tabular} & $93(69.4 \%)$ & $46(57.5 \%)$ & $0(0 \%)$ & $11(61.1 \%)$ & $6(60 \%)$ & $3(75 \%)$ \\
\hline \begin{tabular}{|l|} 
Piperacillin- \\
Tazobactam \\
\end{tabular} & $100(74.6 \%)$ & $51(63.8 \%)$ & $22(91.7 \%)$ & $11(61.1 \%)$ & $8(80 \%)$ & $4(100 \%)$ \\
\hline Meropenem & $119(88.8 \%)$ & $72(90 \%)$ & $23(95.8 \%)$ & $16(88.9 \%)$ & $8(80 \%)$ & $4(100 \%)$ \\
\hline \begin{tabular}{|l|} 
Norfloxacin \\
\end{tabular} & $14(10.4 \%)$ & 15(18.8\%) & $0(0 \%)$ & $2(11.1 \%)$ & $2(20 \%)$ & $0(0 \%)$ \\
\hline Nitrofurantoin & $134(100 \%)$ & $79(98.8 \%)$ & $0(0 \%)$ & $18(100 \%)$ & $10(100 \%)$ & $4(100 \%)$ \\
\hline \begin{tabular}{|l|} 
Ceftazidime \\
\end{tabular} & $1(0.7 \%)$ & $0(0 \%)$ & $21(87.5 \%)$ & $0(0 \%)$ & $0(0 \%)$ & $0(0 \%)$ \\
\hline \multicolumn{7}{|c|}{ Table 5. Antibiotic Sensitivity of Gram-Negative Isolates } \\
\hline
\end{tabular}

A total of 550 samples were collected during the study period and of this 284 were found to be culture positive. Out of this 298 were females and 252 males. In this study 136 males and 148 females were found to be culture positive.

\section{DISCUSSION}

A total of 550 samples were collected during the study period and of this 284 were found to be culture positive. Out of this 298 were females and 252 males. In this study 136 males and 148 females were found to be culture positive. Of the total samples processed, positivity was higher in female $52.1 \%$ than males (47.9\%). In studies conducted by B V Ramana et $\mathrm{al}^{7}$ female patients $(40 \%)$ than males $(30 \%)$, Goutam $\mathrm{R}$ et $\mathrm{al}^{8}$ female (43\%) and males (30\%). According to chi-square test, $p=0.314 .0 .05$, there is no statistically significant association between sex and isolates.

Age- wise distribution of 372 samples belongs to $41-60$ age group, 159 in 61-80 years and 15 in 21-40 years. Only three from age group $>80$ years and one from 18 - 20 years. According to chi=square test, $p=0.141>0.05$, was found that no statically significant association between age and isolates. Dr. B. Murali Krishna, Dr K. S. K Chaitanya Varma and Dr T Srujanitha from Andhra Pradesh found that there is a significant correlation between metabolic control of diabetes and asymptomatic bacteriuria in women with diabetes. This means that significant impairment of metabolic control of the disease increases the risk of developing asymptomatic bacteriuria. ${ }^{9}$

Macroscopy study showed turbid urine in 168, slightly turbid in 53 and clear in 63. The association between significant bacteriuria and macroscopic study showed that the relation is statistically significant $(p=0.000<0.001)$. Significant pyuria was present in 224/284 samples with significant bacteriuria. $P$ value of association between number of pus cells in urine to significant bacteriuria is $0.000(<0.001)$. Thus, there is statistically significant association between pyuria and significant bacteriuria. Wet film for pyuria was $78.87 \%$ sensitive and $78.95 \%$ specific with positive predictive value $80 \%$ and negative predictive of $77.78 \%$. This is similar to the study Tamng et al 2017.10 They found that the higher number of pus cells in urine in the positive culture results was statistically significant. Gram stain has sensitivity of $94.72 \%$ and specificity of $83.46 \%$ and positive predictive value of $85.95 \%$ and negative predictive value of $93.67 \%$. According to this study gram stain was the most sensitive test with high probability that gram stain will be positive in those with significant bacteriuria.

Gram stain also gives immediate information about the nature of infecting organisms and helps to guide in empirical antimicrobial therapy. So, gram stain can be used as good screening test. ${ }^{11}$ In this study, Gram stain correlated with culture in 269 samples. Only 15 samples which showed no organisms in gram stained smear yielded isolate in culture. In culture, significant bacteriuria was obtained in 284 (51.6\%), mixed bacterial growth $60(10.9 \%)$ and no significant bacteriuria in 29 (5.3\%). There is no statistically significant association between duration of diabetes $(p=0.212>0.05)$, treatment taken (p-0.206>0.05), status of diabetes (p$0.115>0.05$ ) and isolates. Of the 284 bacterial isolates $95.1 \%$ were gram negative and gram positive accounted for only $4.9 \%$. This is in accordance with most of the studies by Gautam $\mathrm{R}$ et al, 46 Ramana et al,7 Tamang et al. ${ }^{10} \mathrm{E}$. coli was the most common pathogen isolated in this study and it accounted for about $47.2 \%$. This is similar to other studies from other parts of India and also from different countries across the world. The second common pathogen was Klebsiella species, which contributed to $28.2 \%$ of cases. This is similar to the study by Osanyinpeju Oluwaseum Samuel et al in 2014 in Nigeria E. coli in $45.5 \%$ cases and Klebsiella spp. in $26.2 \%$ cases of urinary tract infection in diabetic patients presented to the health centre and other clinics in Ago-Iwoye, Ogun state Nigeria. ${ }^{12}$ Similar study done by P. Preethishree et al in 2016, reported $E$. coli in $57.14 \%$ and Klebsiella spp in $17.62 \%$ of patients attending tertiary care teaching hospital, Karanataka. ${ }^{13}$ This is also similar to the study done by Gautam R et al ${ }^{8}$ in 2013 . They reported E. coli $(56.75 \%)$ and Klebsiella spp. (38\%) in patients attending out -patients departments at Kasturba Medical College Teaching Hospital, Manipal, India ${ }^{8}$

The third common isolate was Pseudomonas aeruginosa (4.4\%, 24/284). This is also similar to studies of Mohammed et al ${ }^{14}$ in $2016(14.2 \%)$, B. Natesan et al $(11.6 \%)^{15}$ Balamuruganvelu et al $(7.3 \%)^{16}$ and $14.2 \%$ patients attending at Basrah hospital, Iraq. ${ }^{14}$ Acinetobacter baumannii was isolated in 6.3\%. Almost similar study of Manik C. Shill et al in $2010^{17}$ reported Acinetobacter baumannii as the fourth most common isolate in Diagnostic centres in Dhaka. ${ }^{10}$ I solates of Proteus were 10 contribute to $3.5 \%$ isolates. Of this five Proteus vulgaris and five Proteus mirabilis. Gram positive organism isolates were 14 number and of this 13 were Enterococci and only one Methicillin resistant Staphylococcus aureus. Among the 134 isolates of E. coli, all were resistant to Ampicillin. 18.7\% of the isolates were sensitive to Gentamicin and $61.2 \%$ to Amikacin. $3.7 \%$ were sensitive to $1^{\text {st }}$ generation Cephalosporin. $51.5 \%$ were sensitive to Trimethoprimsulfamethoxazole. $12.7 \%$ were sensitive to $3^{\text {rd }}$ generation Cephalosporin. $69.4 \%$ were sensitive to third generation Cephalosporin Cefoperazone sulbactam. $10.4 \%$ sensitive to Norfloxacin and $11.9 \%$ sensitive to Ciprofloxacin. $88.8 \%$ sensitive to Meropenem. $74.6 \%$ of them was sensitive to Piperacillin-tazobactam. $100 \%$ sensitive to Nitrofurantoin. Getnet B et al 18 also reported $100 \%$ resistance to Ampicillin 
and low level resistance to third generation Cephalosporin and Gentamicin. Barad et al also reported high level resistance of $E$ coli towards Ampicillin, Ciprofloxacin, Gentamicin and Cefotaxime. ${ }^{19}$ In another study by Maharj an et al also reported that $E$. coli was found to be highly susceptible to Nitrofurantoin (90.4\%) followed by Amikacin (81\%) as Amoxycillin showed the least sensitivity towards E. coli. ${ }^{20}$

In the present study Klebsiella spp. was found to be more resistant than E. coli. Only $15 \%$ were sensitive to third generation cephalosporin Cefotaxime and $57.5 \%$ sensitive to third generation cephalosporin Cefoperazone- sulbactam. 18.8\% sensitive to both Norfloxacin and Ciprofloxacin. 63.8\% sensitive to Piperacillin- tazobactam. Of the 34 isolates resistant to $3^{\text {rd }}$ generation Cephalosporin. $8.75 \%$ of Klebsiella isolates were found to be ESBL producers. $90 \%$ were sensitive to Meropenem and $98.8 \%$ to Nitrofurantoin.

In this study, Pseudomonas were $25 \%$ sensitive to Gentamicin, $58.3 \%$ sensitive to Amikacin, $33.3 \%$ sensitive to Ciprofloxacin, $87.5 \%$ to Ceftazidime, $91.7 \%$ to Piperacillintazobactam, $95.8 \%$ to Meropenem. Mohammmed et al reported that Pseudomonas were 31\% to Amikacin, $24 \%$ to Ciprofloxacin, $64 \%$ to Piperacillin.

All isolates of Acinetobacter resistant to Ampicillin and $1^{\text {st }}$ generation Cephalosporin. 27.8\% sensitive to Gentamicin, $50 \%$ sensitive to Amikacin, $33.3 \%$ to Trimethoprimsulfamethoxazole. $11.1 \%$ to Norfloxacin and Ciprofloxacin. $16.7 \%$ to third generation cephalosporin Cefotaxime and $61.1 \%$ sensitive to third generation cephalosporin Cefoperazone sulbactam. $61.1 \%$ sensitive to Piperacillintazobactam, $88.9 \%$ to Meropenem and $100 \%$ sensitive to Nitrofurantoin. Manik C Shill et al reported that Acinetobacter were resistance to Ampicillin and Gentamicin. Sensitive to Amikacin and Meropenam. ${ }^{17}$ All isolates of Proteus were 70\% sensitive to Trimethoprim- sulfamethoxazole and 100\% sensitive to Piperacillin-tazobactam, Meropenem and Nitrofurantoin. Enterococci and MRSA are 100\% sensitive to Amikacin and Vancomycin.

\section{Limitations of Study}

Subjects with significant bacteriuria were initiated appropriate treatment after obtaining a second sample for confirmation by the concerned physician. Patients were followed up after one week of stopping antibiotic therapy with a repeat urine culture and sensitivity testing. Almost all patients were followed as much as possible during the study period.

\section{CONCLUSIONS}

Prevalence of urinary tract infection is higher in females compared to males in diabetic patients. Age, duration of diabetes, and treatment taken for diabetes were not found to be associated with significant bacteriuria. Gram stain was found to be the best screening test with high sensitivity and specificity. In our setting, urine microscopy is usually used as a screening test and further urine culture is done only if wet film is positive. But in our study we found that wet film has comparatively low sensitivity and specificity. Majority of the macroscopically turbid urine showed significant bacteriuria in culture. So, it becomes essential that culture should be done for all samples in diabetes to decrease the morbidity and prevent the occurrence of complications.

The predominant pathogens isolated in our study were gram negative bacilli rather than gram positive organisms. $E$. coli was the most common organism isolated followed by Klebsiella spp.

E. coli, the most common organism isolated was comparatively resistant to the commonly used antibiotics like ampicillin and cephalexin. Nitrofurantoin was another oral formulation which was found to be effective for most of the gram negative isolates obtained. Resistance to ampicillin, cephalexin, ciprofloxacin, norfloxacin, trimethoprimsulfamethoxazole observed in these gram-negative isolates may be attributed to the increase in prescribing of these drugs for uncomplicated UTI and other indications in recent years. Complications of UTI in diabetes was not detected in our study, as in most of them diabetes was controlled with insulin, OHA or both and prompt treatment with correct antibiotics.

Authors thank the Department of Microbiology and Diabetic Clinic Govt. Medical College, Kottayam, for allowing them to conduct the study.

Financial or Other Competing Interests: None.

\section{REFERENCES}

[1] Fawwad A, Sabir R, Riaz M, et al. Uropathogens and the Antimicrobial susceptibility patterns in patients with type 2 diabetes. Austin Journal of Endocrinology and Diabetes 2014;1(5):1-4.

[2] Leslie C, Albert B, Max S. Topley and Wilson's Microbiology and microbial infections. Vol. 3. $10^{\text {th }}$ edn. London: Edward Arnold 1998: p. 672-80.

[3] Mandell GL, Bennett JE, Dolin R. Urinary tract infections. Mandell, Douglas and Bennett's Principles and practice of infectious diseases. $7^{\text {th }}$ edn. Churchill Livingstone 2009: p. 957-76.

[4] Forbes BA, Sahm DF, Weissfeld AS. Infections of urinary tract. Bailey and Scott's Diagnostic Microbiology. $12^{\text {th }}$ edn. Missouri: Mosby Elsevier 2007: p. 842-54.

[5] Winn W Jr, Allen S, Janda W, et al. Koneman's colour atlas and textbook of Diagnostic Microbiology. $6^{\text {th }}$ edn. Lippincott Williams \& Wilkms 2006: p. 82-670.

[6] CLSI. Performance Standards for Antimicrobial Susceptibility testing, 28th edition. CLSI Supplement M100. Wayne, PA: Clinical and Laboratory Standards Institute 2018;38(3).

[7] Ramana BV, Chauhury A. Prevalence of uropathogens in diabetic patients and their resistance pattern at a tertiary care centre in South India. Int J Biol Med Res 2012;3(1):1433-5.

[8] Gautam R, Acharya A, Nepal HP, et al. Urinary tract infections in diabetic patients. World Journal of Pharmacy and Pharmaceutical Science 2013;2(3):1146-56.

[9] Muralikrishna B, Varma KSKC, Srujanitha T. Asymptomatic bacteriuria in women with diabetes mellitus. Int J Sci Res 2017;6(6):29-31.

[10] Tamang K, Shrestha P, Koirala A, et al. Prevalence of bacterial uropathogens among diabetic patients 
attending Padma nursing hospital of Western Nepal. Himalayan Journal of Science and Technology 2017;1(1):15-9.

[11] Patil SS, Mahale K, Krishna S, et al. Evaluation of gram stain of uncentrifuged urine as a screening method for diagnosis of urinary tract infections. Current Research in Medicine and Medical Sciences 2011;1(2):19-21.

[12] Samuel 00, Mathew AO, Mopelola DA, et al. Asymptomatic urinary tract infection in diabetic patients in Ago-Iwoye, Ogun State, Nigeria. Journal of American Science 2014;10(Suppl 4):72-8.

[13] Preethishree P, Rai R, Kumar VK, et al. Uropathogens and their antibiotic susceptibility pattern at a tertiary care teaching hospital in Coastal Karnataka, India. Int J Curr Microbiol App Sci 2016;5(1):23-31.

[14] Mohammed AAI. Incidence and sensitivity of bacterial uropathogens among diabetic patients. European Journal of Experimental Biology 2016;6(3):8-12.

[15] Natesan B, Banu T. A study on bacteriological profile of urinary tract infection in diabetes mellitus patients in a tertiary care hospital. Int J Curr Microbiol App Sci 2017;6(7):2577-83.
[16] Balamurganvelu S, Geethavani B, Reddy SV, et al. Drug resistance pattern of uropathogens causing asymptomatic bacteriuria. African Journal of Clinical and Experimental Microbiology 2017;18(3):145-53.

[17] Shill MC, Huda NH, Moain FB, et al. Prevalence of uropathogens in diabetic patients and their corresponding resistance pattern. Results of a survey conducted at diagnostic centres in Dhaka, Bangladesh. Oman Med J 2010;25(4):282-5.

[18] Getenet B, Wondewosen T. Bacterial uropathogens in urinary tract infection and antibiotic susceptibility pattern in Jimma university specialised hospital, southwest Ethiopia. Ethiop J Health Sci 2011;21(2):1416.

[19] Barad PS, Barate DL. Prevalence of antibiotic susceptibility pattern of bacteria isolated from catheter associated urinary tract infection. Bioscience Discovery 2018;9(1):1-7.

[20] Maharjan MN, Mandal KP, Sharma KV. Comparative study among the bacterial causes of urinary tract infection in diabetic and non-diabetic patients visiting Alka Hospital, Lalitpur. Ann Clin Med Microbiol 2015;1(2):1006. 